\title{
Common iatrogenic pleural complications
}

\author{
Amelia O. Dunscombe • Nick A. Maskell
}

Published online: 29 March 2012

(C) Springer Science+Business Media, LLC 2012

\begin{abstract}
All invasive pleural procedures have the potential to cause harm. Complications from pleural procedures include empyema, intercostal artery laceration, hemothorax, and pneumothorax as well as other organ puncture. Many of these complications will be life threatening and will increase morbidity and hospital length of stay. An understanding of iatrogenic pleural disease helps clinicians to appreciate how these risks can be minimized and complications managed promptly and effectively. This review systematically evaluates the current evidence and guidelines regarding iatrogenic pleural complications and their management. Whilst impossible to eliminate procedural risk entirely, complications will be reduced to a minimum by ensuring adequate medical training, use of pleural ultrasound, and adherence to guidelines and standard operating procedures (SOPs).
\end{abstract}

Keywords Pleural effusion - Pneumothorax - Iatrogenic . Thoracentesis · Chest drain · Complication · Pleural disease . Thoracoscopy

\section{Introduction}

Invasive pleural procedures, including simple pleural fluid aspiration, thoracentesis, and chest drain insertions are commonly undertaken to aid the diagnosis and management of a variety of pleural diseases. Although in the vast majority of

A. O. Dunscombe $\cdot$ N. A. Maskell ( $\square)$

Academic Respiratory Unit, School of Clinical Science,

University of Bristol, Southmead Hospital,

Bristol BS10 5NB, UK

e-mail: nick.maskell@bristol.ac.uk

A. O. Dunscombe

e-mail: amelia.dunscombe@nbt.nhs.uk cases they occur safely, there are many reports of serious complications following these procedures [1]. These can be minimized by image guidance and adequate training $[2,3 \cdot \bullet, 4 \cdot]$.

The development of newer techniques, such as local anaesthetic thoracoscopy [5], and the insertion of indwelling pleural catheters has advanced the investigation and management of pleural disease. However, with the advent of these techniques, a thorough understanding of the procedural risks to patients is imperative to inform clinical decisions and provide guidance on how complications should be managed if they occur.

Given the close proximity of the pleura to structures in the neck and abdomen, pleural complications can also be caused by extra thoracic interventions, such as abdominal surgery and central venous catheter insertion. In addition, breach of the pleura during transthoracic lung biopsy may result in pleural complications and barotrauma secondary to mechanical ventilation is a well-known cause of iatrogenic pneumothoraces.

This review will evaluate the potential iatrogenic pleural complications and examine the current literature and guidelines on the subject.

\section{Iatrogenic pneumothorax}

Iatrogenic pneumothoraces are a common complication of a variety of interventions and the literature would suggest they occur more commonly than spontaneous pneumothoraces [6]. They can be induced by thoracic procedures, including transthoracic biopsies, transbronchial lung biopsy, thoracentesis, chest drain insertion or pacemaker insertion, or they can result from procedures involving the neck, such as central venous catheter insertion, or the abdomen, as well 
as barotrauma to the pleura from invasive ventilation $[7,8]$. It can be associated with significant morbidity and mortality and may prolong the length of hospital stay, particularly if related to critical illness [9].

A number of potential mechanisms can contribute to the development of iatrogenic pneumothoraces. Puncture of the visceral pleura and lung during an invasive procedure can result in an air leak into the pleural cavity from the lung itself, or alternatively air can be entrained through the device being used for the procedure from the outside. Shearing forces on the pleural surface created during lung re-expansion in patients with pleural adhesions may result in a visceral pleural tear and subsequent pneumothorax developing.

In patients with underlying trapped lung, whereby a visceral pleural rind prevents lung re-expansion following the removal of pleural fluid, air can be sucked into the pleural cavity due to the more negative intrapleural pressure created by the less elastic visceral pleura [10••]. This results in an unavoidable pneumothorax, which is commonly termed pneumothorax "ex vacuo." It is a fairly common finding and may occur in those with both pleural malignancy or benign pleuritis [11]. Insertion of a chest drain in this situation is unlikely to be beneficial as expansion of the underlying lung is restricted.

The incidence of iatrogenic pneumothorax varies depending upon the procedure performed and is particularly common following thoracentesis and central venous cannulation [7].

Pneumothoraces following thoracentesis are a relatively common finding and have been reported to occur in between $0 \%$ and $19.2 \%$ of patients [12•]. A recent meta-analysis found a number of potential risk factors, including an inexperienced operator performing the procedure, the use of a large bore needle, therapeutic thoracentesis as opposed to a diagnostic tap, witnessed aspiration of air during the procedure, multiple aspiration attempts, and concurrent mechanical ventilation [12•]. Large volume pleural aspirations may result in a higher risk of procedure-related pneumothorax, particularly if more than $1.8 \mathrm{~L}$ is aspirated in a single procedure [13]. A study by the Mayo Clinic, whereby improvements in training and the use of ultrasound guidance in pleural aspiration were instituted, found the iatrogenic pneumothorax rate was reduced from $8.6 \%$ to $1.1 \%$ with these interventions $(P=0.0034)\left[4^{\bullet}\right]$.

Transthoracic lung biopsies may also result in the development of a pneumothorax. A recent large, populationbased, cross-sectional analysis of 15,865 patients undergoing transthoracic needle lung biopsy for pulmonary nodules in four US states, found an overall pneumothorax risk of $15 \%$ (95\% CI $14.0 \%$ to $16.0 \%$ ), with $6.6 \%$ (95\% CI $6.0 \%$ to $7.2 \%$ ) of patients in the study requiring a chest tube to be inserted to treat a pneumothorax [14•]. A UK survey of 5444 percutaneous lung biopsies showed a pneumothorax risk of $20.5 \%$, with $3.1 \%$ of patients in the survey requiring a drain insertion [15].
However, predicting which patients will develop a pneumothorax in this setting is difficult. The presence of COPD may be a risk factor $[14 \cdot, 16,17]$ as well as a smaller needlepleural angle when taking the biopsy $[18,19]$. Some studies suggest that the deeper the lesion being biopsied, the higher the risk of pneumothorax [17, 19], but this finding is not consistent across all studies $[18,20]$. CT findings alone do not appear to be an accurate predictor [21].

Closed pleural biopsies also confer a risk of iatrogenic pneumothorax of between $0 \%-5 \%$ [22, 23]. Image guidance improves the diagnostic yield compared with blind pleural biopsy and may also be associated with fewer complications [24]. After the procedure, patients may be asked to lie on the side of the procedure to minimize the development of air leaks and pneumothorax, although this practice is only anecdotal and a small case control trial did not show it to be of benefit [10••, 25].

Radiofrequency ablation (RFA) of lung tumors is also associated with a significant risk of pneumothorax, as well as aseptic pleuritis [26]. A systematic review of the literature found the iatrogenic pneumothorax rate to be $4.5 \%-61.6 \%$ after RFA, but most cases were self-limiting [27]

Pneumothoraces on the intensive care unit are a common occurrence and can be related to barotrauma from positive pressure ventilation or be secondary to pleural puncture during invasive procedures (such as central line insertion or transbronchial biopsies). They occur in about $3 \%$ of patients admitted to the intensive care unit (ICU) and represent a potentially life-threatening event, particularly if associated with mechanical ventilation [9]. A prospective study of over 3000 patients from 11 French ICUs showed that those who developed a pneumothorax during the first 30 days of their admission were more than twice as likely to die as those who did not [9].

Shearing forces from positive pressure ventilation may result in the development of a bronchopleural fistula, which can quickly enlarge due to the continuous pressure from the ventilator and may result in a tension pneumothorax. Therefore, all patients with a pneumothorax in the context of mechanical ventilation require formal chest drainage to minimize the risk of a tension pneumothorax developing, and lung-protective ventilation strategies may also be helpful.

Given the dangers of pneumothoraces in the context of mechanical ventilation, there may be reluctance to drain effusions in this patient population. However, a recent meta-analysis evaluating the risks and benefits of draining pleural effusions in mechanically ventilated patients, showed that pleural fluid drainage improved oxygenation and may confer benefits with regard to respiratory dynamics in these patients. The pooled risk of pneumothorax was $3.4 \%$ (95\% CI $1.7 \%$ to $6.5 \%$ ) [28]. In order to minimize the risk of pneumothorax when inserting a chest tube in a 
patient on mechanical ventilation, the positive endexpiratory pressure (PEEP) should be turned off and the ventilator briefly disconnected as the drain is inserted [29].

Aside from iatrogenic pneumothoraces in the critical care setting, the management will depend on the size of the pneumothorax, the degree of patient's symptoms, and their comorbidities. The majority will resolve spontaneously by observation alone and in those who require an intervention, simple aspiration is usually sufficient [6]. A small proportion of patients may require chest tube drainage, and this is more likely in patients with underlying COPD [20].

However, patients who develop a pneumothorax in the context of mechanical ventilation do require a chest drain to be inserted to minimize the risk of a tension pneumothorax developing.

\section{Subcutaneous emphysema}

In severe cases of iatrogenic pneumothorax, whereby a large visceral pleural defect results in the creation of a bronchopleural fistula, air can be forced into the tissues of the chest wall, resulting in the development of subcutaneous emphysema. It may also occur if a chest drain becomes displaced and one or more of the drainage holes in the tube migrates into the subcutaneous tissues.

This initially results in localized thoracic swelling and crepitus on palpation, or may only be identified on chest radiography. Subsequently, the air may spread along fascial planes to become more generalized. In severe cases, the neck and face can become swollen, which may result in airway compromise [10•*]. In a recent UK series of 824 chest drains inserted for a variety of indications, $3.4 \%$ of patients developed surgical emphysema as a complication $[30 \bullet]$.

If a chest drain is already in situ, it should be carefully evaluated to ensure it is correctly positioned. If not, a drain should be inserted to treat the pneumothorax and in many cases this will prevent the surgical emphysema deteriorating. However, a small chest drain may be inadequate to keep up with a large air leak and hence a larger bore tube, or multiple chest tubes may be required. Use of thoracic suction may also be beneficial in such cases. Liaison with the thoracic surgeons regarding definitive surgical treatment of the bronchopleural fistula may become necessary.

\section{Iatrogenic pleural infection}

Invasive procedures create a tract whereby infection can enter the soft tissues and pleural cavity. Chest drain insertions carry a $2 \%-3 \%$ risk [31], but this is increased in the context of trauma [32]. Empirical antibiotics have been shown to be beneficial in reducing the incidence of pleural infection when a chest drain is inserted for trauma $[33 \bullet, 34]$; however, outside this context, there have been no clinical trials evaluating their use and they are not routinely given.

Making the diagnosis of iatrogenic pleural infection is the same as for other clinical contexts [35]. Fluid and blood should be sent for culture to help identify a causative organism and identify antibiotic resistance. Fluid drainage, antibiotics, and careful attention to nutrition are key components of management. Antibiotics should be broad spectrum and have good cover for hospital-acquired organisms, such as the gram negatives and methicillin-resistant Staphylococcus aureus (MRSA). If a chest drain becomes secondarily infected, it may be necessary to remove the infected drain and re-site a new one. Some patients may require surgical intervention if medical management fails.

Indwelling pleural catheters are increasingly used in the management of malignant pleural disease to allow intermittent pleural drainage in the community, but do confer an increased risk of pleural infection and localized chest wall cellulitis as they remain in situ for long periods of time [36]. Strict asepsis at the time of drain placement, along with careful patient education regarding drain care, may help to reduce the risk of infection.

Currently the rate of pleural infection in indwelling pleural catheters in the literature is $2 \%-12 \%$ [37-40]. In addition, as with any implanted device, colonization of the catheter may occur, particularly by coagulase-negative Staphylococcus [41], although the clinical relevance of this in causing subsequent infection is not clear.

\section{Intrapleural hemorrhage}

Due to the anatomy of the intercostal neurovascular bundle, pleural procedures confer a risk of intrapleural hemorrhage if these vessels are lacerated. The neurovascular bundle may be protected by the subcostal groove of the rib above; however, as the intercostal nerves and vessels travel more posteriorly, they become exposed, lying in the middle of the intercostal space, rather than being tucked under the rib. In addition, the intercostal collateral artery lies above the rib and is also vulnerable to damage [42]. Pleural procedures should therefore be performed in as lateral a position as possible and above a rib to try and prevent neurovascular damage $[3 \bullet \cdot$. Some advocate using an area $30 \%-50 \%$ above the rib to also minimize the risk of damaging the intercostal collateral artery [42]. Figure 1 shows an intercostal artery laceration caused by a simple diagnostic pleural fluid aspiration using a posterior approach.

Rates of bleeding following pleural procedures are low however and range from $0 \%-2 \%$ in the literature but depend on the type of thoracic procedure performed $[10 \bullet \cdot$. 
Fig. 1 A CT scan of a patient with a hemothorax caused by laceration of an intercostal artery during a diagnostic pleural aspiration performed with a posterior approach.

Extravasation of CT contrast from the injured vessel into the pleural cavity can be seen
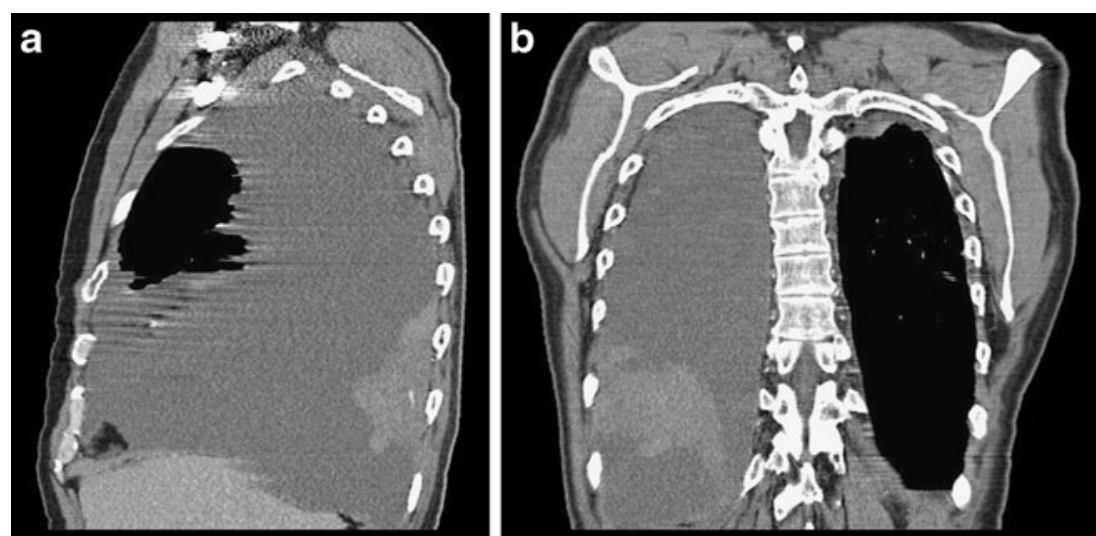

Any coagulopathy or platelet deficiency should be corrected prior to invasive pleural procedures in order to minimize the risk of intrapleural hemorrhage [3••].

Departments performing pleural interventions should have a Standard Operating Procedure (SOP) in place regarding the management of intrapleural hemorrhage. Resuscitation is paramount in the first instance and once the patient is stabilized, definitive treatment to stem the bleeding from the vessel is required. This can be done radiologically or surgically, depending on the patient's condition and local availability. An SOP template for the management of iatrogenic pleural hemorrhage can be found in Table 1, but would need to be adapted for use at individual centers where infrastructure may vary.

\section{Drain malposition}

Insertion of a chest drain may be indicated in a variety of clinical scenarios, including the management of symptomatic pleural effusion, pleural infection, and pneumothorax. Chest drains may be inserted using blunt dissection or a Seldinger technique, but insertion using a trochar is no longer recommended due to an increased risk of trauma to surrounding structures [3••]. The use of small-bore Seldinger drains has become very widespread [3••], and a recent study evaluating patients with pleural infection suggests that smaller drains are less painful than larger ones and are potentially as efficacious [43•]. However, as with all pleural procedures, the insertion of chest drains is not without potential complications (Table 2).

Malposition of drains can be associated with severe morbidity and mortality and was identified as a serious issue by the National Patient Safety Agency in 2008 [1]. They identified 12 deaths and 15 cases of serious harm to patients caused by chest drain insertion in the UK, which had been reported between January 2005 and March 2008 and several other cases of poor chest drain management [1]. This has led to the introduction of measures to improve the safety of these procedures with regard to improvement in training, guideline development, redesign of the equipment used, and regular audit of the activity [30•].
In 2008, a survey of UK acute hospital trusts regarding chest drain complications in the preceding 5 years was undertaken. A total of 67 responding trusts reported major complications, including lung or chest wall injury (47 cases), wrong side procedures (6 cases), lost guidewires (3 cases), and drain misplacement (31 cases). Eight deaths were reported as a result of these complications [44].

The British Thoracic Society undertook a national audit of chest drain practice in the UK, which included 824 drains inserted during the 2-month audit period. This also highlighted a number of potential complications, including pain after the procedure (18\%), drains falling out prematurely $(7.3 \%)$, drain malposition $(2.4 \%)$, and drains becoming blocked (7.4\%) [30•].

Placement of the drain into the lung parenchyma itself has been reported in up to $10 \%$ of drain insertions, although the incidence varies between studies given different insertion techniques and availability of radiological guidance $[45,46]$.

On occasion, the drain may inadvertently lie in a lung fissure, which may result in inefficient drain function. In one French study, evaluating the position of 122 chest drains using CT on a surgical ICU, intrafissural drain placement was found to be as high as $21 \%$ and occurred more commonly with right-sided drains due to the anatomy of the horizontal fissure when chest drains are inserted in the safe triangle [46]. However, the relevance of an intrafissural position is debated and drains should only be re-sited if they are not functioning correctly.

Rarely, drains can accidently perforate other structures, including the diaphragm, heart, stomach, mediastinum, thoracic duct, major vessels, and intra-abdominal organs, with occasionally catastrophic consequences [47]. Direct radiological guidance when inserting chest drains helps to delineate the anatomy of nearby structures and hence minimize the chances of this occurring.

Other rare complications of chest drain placement have been reported including inadvertent compression of vessels or nerves, resulting in pressure effects, including Horner's syndrome [48] and cardiogenic shock from compression of the right ventricle [49]. 
Table 1 A template standard operating procedure for the management of iatrogenic pleural hemorrhage

Standard operating procedure for the management of iatrogenic pleural hemorrhage

Evaluate need for immediate resuscitation:

Has the patient suffered, or do they appear close to cardiac arrest? Or, Are they suffering from severe hypotensive shock? Or, Is the bleeding very severe/torrential?

Yes:

1. Summon help from senior doctor, nursing staff, and/or cardiac arrest team as necessary

2. Initiate standard cardiopulmonary resuscitation if needed

3. Establish large bore venous access and initiate immediate large volume fluid replacement with plasma expander/blood

4. Urgently cross match 4 units of blood

5. Record blood pressure, heart rate, and oxygen saturations every 5 mins

6. Contact cardiothoracic surgeon and the intensive care team to arrange rapid transport to a suitable environment for emergency surgery No:

1. Summon help of senior doctor and nursing staff

2. Apply direct external pressure to pleural puncture site if possible

3. Establish large bore venous access

4. Record blood pressure, heart rate, and oxygen saturations every 10 mins (or more often if required)

5. Initiate immediate large volume fluid replacement with plasma expander/blood if necessary

6. Urgently cross match 4 units of blood

7. If the patient becomes hemodynamically unstable or the bleeding becomes torrential, be ready to summon the cardiac arrest team and initiate standard cardiopulmonary resuscitation; if so, follow steps outlined above

8. Contact local respiratory consultant as a matter of urgency

9. Make personal contact with a senior interventional radiologist to begin planning for imaging and emergency intercostal artery embolization if needed

10. If embolization is not possible or unavailable, contact cardiothoracic surgical team to plan for surgery if required

11. Stop all anticoagulants, including heparin for DVT prophylaxis

In order to minimize drain malposition, pleural ultrasound is recommended to guide all pleural procedures for effusions $[3 \bullet \bullet$. This allows direct visualization of the appearance and depth of the pleural fluid and may identify unforeseen features, such as loculations, a raised hemidiaphragm, or close proximity of other organs (for example the heart, liver, or spleen) which will alter the selection of a site for drain insertion. Similarly, on-table ultrasound should be used prior to local anaesthetic thoracoscopy to guide the selection of a suitable site for the port.

As a result of this, there has been a dramatic increase in the numbers of non-radiology trained physicians performing thoracic ultrasound to guide pleural procedures. With suitable training, this has been shown to be safe and effective [50]. National bodies such as the Royal College of Radiologists in the UK and the Australasian Society for Ultrasound in Medicine offer guidance and training syllabuses for performing thoracic ultrasound and it is important that clinicians undertaking ultrasound are suitably certified [51, 52]. However, despite the evidence for using ultrasound in this context, there is still a lack of availability. Only $52 \%$ of the chest drains included in the 2010 BTS audit were inserted using bedside ultrasound guidance [30•].
In some scenarios where the anatomy is difficult to delineate even with ultrasound, CT may be required to guide the drain insertion. On occasion, CT can also be useful when a drain has been inserted but is not functioning correctly to identify the anatomical location of the drain tract and guide subsequent management.

\section{Malignant seeding}

In the context of underlying pleural malignancy, in particular malignant pleural mesothelioma, invasive pleural procedures may cause seeding of tumor cells along the tract created during the intervention. This may result in the development of painful tumor deposits at the procedure site, known as procedure tract metastases (PTM), which can be difficult to treat once they develop. The larger the incision made in the chest wall, the higher the chance of tract metastases developing [53].

There is a significant time lag between the pleural intervention and the development of tract metastases. The timing varies greatly between studies, but a recent review of the literature found the mean time to development of PTM was 6.5 months (range 4.5-9 months) [54]. 
Prophylactic radiotherapy may be given to patients with mesothelioma who undergo invasive pleural procedures to try and prevent this occurring; however, its benefit is debated [55•]. Three randomized trials have been conducted to evaluate the role of prophylactic radiotherapy in mesothelioma; however, all were underpowered and the results were conflicting [56-58].

Tract metastases have also been reported in the context of indwelling pleural catheters, caused by both mesothelioma and adenocarcinoma [59]. Again the role for prophylactic radiotherapy in this context is not known.

\section{Pleural complications from abdominal procedures}

Procedures involving the abdomen can also result in diaphragmatic and pleural puncture and subsequent pleural complications. In our own center, a percutaneous drain insertion into a liver abscess resulted in pleural infection as the drain transected the diaphragm into the pleural cavity.

Retroperitoneal surgical procedures may expose the parietal pleura and thereby risk breaching the pleural surface. In a case series of 91 radical nephrectomies, 58 were complicated by a pleural injury and this was associated with a prolonged length of hospital stay, particularly in those who had a postoperative chest drain placed [60].

Diaphragmatic and pleural injury is also a complication of laparoscopic renal surgery, with an incidence of $0.6 \%$ in one large series [61]. Due to the use of pressurized intraabdominal carbon dioxide during laparoscopy to improve visualization of the abdominal structures, diaphragmatic injury during the procedure may lead to a rapidly developing pneumothorax or pneumomediastinum with subsequent subcutaneous emphysema. Hence early recognition and repair is paramount.

A French study evaluating respiratory complications after 112 hepatectomies of living liver donors found the incidence of postoperative pleural effusion on CT at 7 days to be as high as $75 \%$ and was particularly high in those who had the right lobe of the liver removed. Empyema developed in three cases $(2.7 \%)$ and pneumothorax in three cases (2.7\%) [62].

Peritoneal dialysis is commonly used for renal replacement therapy in those with end-stage renal failure. Dialysate is infused into the peritoneal cavity via a tunnelled catheter and pleural effusions (particularly on the right) are a recognized complication due to the increased intraabdominal pressure in the context of a diaphragmatic defect. The reported incidence is under $2 \%$ of patients having peritoneal dialysis [63] and if persistent may require the patient to use an alternative method for renal replacement therapy. 


\section{Pleural complications from other thoracic procedures}

Due to the close proximity of the pleura to the other structures within the thorax, pleural complications may occur during thoracic procedures, including cardiothoracic and esophageal surgery.

Post-coronary artery bypass grafting (CABG) pleural effusion is a common complication and can occur early ( $<30$ days after the surgery) or late [64]. The prevalence is highest in the immediate post-operative period, but a significant effusion may still be present in around $10 \%$ of patients after 1 month [65]. Often in the case of early post-CABG pleural effusion, symptoms are minimal and most settle without treatment, but if they persist patients may require repeated therapeutic aspirations for symptomatic relief.

Iatrogenic esophageal leaks and perforations can result in the development of pleural effusions, mediastinitis, and pleural infection in the context of esophageal surgery. Minimally invasive esophagectomy may confer a lower risk of post-operative pleural effusion than open procedures, although the need for re-intervention may be higher [66].

Damage to other thoracic structures including the thoracic duct, resulting in a chylothorax and vessel puncture causing a hemothorax, are also potential complications of intrathoracic procedures [67].

\section{Conclusions}

Iatrogenic pleural complications can occur due to a variety of invasive procedures and are associated with significant morbidity and mortality. The increasing use of bedside thoracic ultrasound for pleural procedures, equipment incorporating safety features, and use of protocols and more extensive training, all help to minimize the risks. It is, however, important to recognize that complications cannot be entirely eliminated and standard procedures should be in place to optimize patient management if a complication does occur.

Disclosure No potential conflicts of interest relevant to this article were reported.

\section{References}

Papers of particular interest, published recently, have been highlighted as:

- Of importance

•- Of major importance

1. National Patient Safety Agency. Risks of chest drain insertion. 200815 May 2008]; NPSA/2008/RRR003]. Available from: www.npsa.nhs.uk/patientsafety/alerts-and-directives.
2. Wrightson JM, Fysh E, Maskell NA, et al. Risk reduction in pleural procedures: sonography, simulation and supervision. Curr Opin Pulm Med. 2010;16(4):340-50.

3. •- Havelock T, Teoh R, Laws D, et al. Pleural procedures and thoracic ultrasound: British Thoracic Society Pleural Disease Guideline 2010. Thorax. 2010;65 Suppl 2:ii61-76. The current UK guidelines on pleural procedures, focusing on risk reduction.

4. - Duncan DR, Morgenthaler TI, Ryu JH, et al. Reducing iatrogenic risk in thoracentesis: establishing best practice via experiential training in a zero-risk environment. Chest. 2009;135(5):1315-20. A study demonstrating improved patient safety following institution of specific training and ultrasound guidance for pleural aspirations.

5. Rahman NM, Ali NJ, Brown G, et al. Local anaesthetic thoracoscopy: British Thoracic Society Pleural Disease Guideline 2010. Thorax. 2010;65 Suppl 2:ii54-60.

6. MacDuff A, Arnold A, Harvey J. Management of spontaneous pneumothorax: British Thoracic Society Pleural Disease Guideline 2010. Thorax. 2010;65 Suppl 2:ii18-31.

7. Sassoon CS, Light RW, O'Hara VS, et al. Iatrogenic pneumothorax: etiology and morbidity. Results of a Department of Veterans Affairs Cooperative Study. Respiration. 1992;59(4):215-20.

8. Despars JA, Sassoon CSH, Light RW. Significance of iatrogenic pneumothoraces. Chest. 1994;105(4):1147-50.

9. de Lassence A, Timsit JF, Tafflet M, et al. Pneumothorax in the intensive care unit: incidence, risk factors, and outcome. Anesthesiology. 2006;104(1):5-13.

10. •• Wrightson JM, Helm EJ, Rahman NM, et al. Pleural procedures and pleuroscopy. Respirology. 2009;14(6):796-807. A thorough and comprehensive review of the literature surrounding complications from pleural procedures.

11. Brims FJ, Arif M, Chauhan AJ. Outcomes and complications following medical thoracoscopy. Clin Respir J 2011.

12. - Gordon CE, Feller-Kopman D, Balk EM, et al. Pneumothorax following thoracentesis: a systematic review and meta-analysis. Arch Intern Med. 2010;170(4):332-9. A meta-analysis identifying risk factors for pneumothorax following thoracentesis.

13. Josephson T, Nordenskjold CA, Larsson J, et al. Amount drained at ultrasound-guided thoracentesis and risk of pneumothorax. Acta Radiol. 2009;50(1):42-7.

14. - Wiener RS, Schwartz LM, Woloshin S, et al. Population-based risk for complications after transthoracic needle lung biopsy of a pulmonary nodule: an analysis of discharge records. Ann Intern Med. 2011;155(3):137-44. A large population-based study evaluating the risks of percutaneous lung biopsy.

15. Richardson CM, Pointon KS, Manhire AR, et al. Percutaneous lung biopsies: a survey of UK practice based on 5444 biopsies. $\mathrm{Br}$ J Radiol. 2002;75(897):731-5.

16. Fish GD, Stanley JH, Miller KS, et al. Postbiopsy pneumothorax: estimating the risk by chest radiography and pulmonary function tests. AJR Am J Roentgenol. 1988;150(1):71-4.

17. Poe RH, Kallay MC, Wicks CM, et al. Predicting risk of pneumothorax in needle biopsy of the lung. Chest. 1984;85(2):232-5.

18. Ko JP, Shepard JO, Drucker EA, et al. Factors influencing pneumothorax rate at lung biopsy: are dwell time and angle of pleural puncture contributing factors? Radiology. 2001;218(2):491-6.

19. Hiraki T, Mimura H, Gobara H, et al. Incidence of and risk factors for pneumothorax and chest tube placement after CT fluoroscopyguided percutaneous lung biopsy: retrospective analysis of the procedures conducted over a 9-year period. AJR Am J Roentgenol. 2010;194(3):809-14.

20. Anderson CL, Crespo JC, Lie TH. Risk of pneumothorax not increased by obstructive lung disease in percutaneous needle biopsy. Chest. 1994;105(6):1705-8.

21. Chakrabarti B, Earis JE, Pandey R, et al. Risk assessment of pneumothorax and pulmonary haemorrhage complicating percutaneous coaxial cutting needle lung biopsy. Respir Med. 2009;103(3):449-55. 
22. Benamore RE, Scott K, Richards CJ, et al. Image-guided pleural biopsy: diagnostic yield and complications. Clin Radiol. 2006;61 (8):700-5.

23. Adams RF, Gleeson FV. Percutaneous image-guided cuttingneedle biopsy of the pleura in the presence of a suspected malignant effusion. Radiology. 2001;219(2):510-4.

24. Maskell NA, Gleeson FV, Davies RJ. Standard pleural biopsy versus CT-guided cutting-needle biopsy for diagnosis of malignant disease in pleural effusions: a randomised controlled trial. Lancet. 2003;361(9366):1326-30.

25. Berger R, Smith D. Efficacy of the lateral decubitus position in preventing pneumothorax after needle biopsy of the lung. South Med J. 1988;81(9):1140-3.

26. Kashima M, Yamakado K, Takaki H, et al. Complications after 1000 lung radiofrequency ablation sessions in 420 patients: a single center's experiences. AJR Am J Roentgenol. 2011;197(4):W576-80.

27. Zhu JC, Yan TD, Morris DL. A systematic review of radiofrequency ablation for lung tumors. Ann Surg Oncol. 2008;15 (6): 1765-74.

28. Goligher EC, Leis JA, Fowler RA, et al. Utility and safety of draining pleural effusions in mechanically ventilated patients: a systematic review and meta-analysis. Crit Care. 2011;15(1):R46.

29. Shaikhrezai K, Zamvar V. Hazards of tube thoracostomy in patients on a ventilator. J Cardiothorac Surg. 2011;6:39.

30. - Hooper C, Maskell N. British Thoracic Society national pleural procedures audit 2010. Thorax. 2011;66(7):636-7. A large national audit of chest drain insertion practices in the UK, including 824 drains inserted and the complications encountered.

31. Chan L, Reilly KM, Henderson C, et al. Complication rates of tube thoracostomy. Am J Emerg Med. 1997;15(4):368-70.

32. Bailey RC. Complications of tube thoracostomy in trauma. J Accid Emerg Med. 2000;17(2):111-4.

33. - Bosman A, de Jong MB, Debeij J, et al. Systematic review and meta-analysis of antibiotic prophylaxis to prevent infections from chest drains in blunt and penetrating thoracic injuries. Br J Surg 2011. A systematic review of antibiotic prophylaxis for chest drain insertions in the context of trauma.

34. Fallon Jr WF, Wears RL. Prophylactic antibiotics for the prevention of infectious complications including empyema following tube thoracostomy for trauma: results of meta-analysis. J Trauma. 1992;33((1):110-6. discussion 116-7.

35. Davies HE, Davies RJ, Davies CW. Management of pleural infection in adults: British Thoracic Society Pleural Disease Guideline 2010. Thorax. 2010;65 Suppl 2:ii41-53.

36. Roberts ME, Neville E, Berrisford RG, et al. Management of a malignant pleural effusion: British Thoracic Society Pleural Disease Guideline 2010. Thorax. 2010;65 Suppl 2:ii32-40.

37. Putnam Jr JB, Walsh GL, Swisher SG, et al. Outpatient management of malignant pleural effusion by a chronic indwelling pleural catheter. Ann Thorac Surg. 2000;69(2):369-75.

38. Tremblay A, Michaud G. Single-center experience with 250 tunnelled pleural catheter insertions for malignant pleural effusion. Chest. 2006;129(2):362-8.

39. van den Toorn LM, Schaap E, Surmont VF, et al. Management of recurrent malignant pleural effusions with a chronic indwelling pleural catheter. Lung Cancer. 2005;50(1):123-7.

40. Sioris T, Sihvo E, Salo J, et al. Long-term indwelling pleural catheter (PleurX) for malignant pleural effusion unsuitable for talc pleurodesis. Eur J Surg Oncol. 2009;35(5):546-51.

41. Morel A, Mishra E, Medley L, et al. Chemotherapy should not be withheld from patients with an indwelling pleural catheter for malignant pleural effusion. Thorax. 2011;66(5):448-9.

42. Wraight WM, Tweedie DJ, Parkin IG. Neurovascular anatomy and variation in the fourth, fifth, and sixth intercostal spaces in the midaxillary line: a cadaveric study in respect of chest drain insertion. Clin Anat. 2005;18(5):346-9.
43. - Rahman NM, Maskell NA, Davies CW, et al. The relationship between chest tube size and clinical outcome in pleural infection. Chest. 2010;137(3):536-43. A study of pleural infection, suggesting small drains are as effective and less painful.

44. Harris A, O'Driscoll BR, Turkington PM. Survey of major complications of intercostal chest drain insertion in the UK. Postgrad Med J. 2010;86(1012):68-72.

45. Stark DD, Federle MP, Goodman PC. CT and radiographic assessment of tube thoracostomy. AJR Am J Roentgenol. 1983;141 (2):253-8.

46. Remerand F, Luce V, Badachi Y, et al. Incidence of chest tube malposition in the critically ill: a prospective computed tomography study. Anesthesiology. 2007;106(6):1112-9.

47. Meisel S, Ram Z, Priel I, et al. Another complication of thoracostomyperforation of the right atrium. Chest. 1990;98(3):772-3.

48. Levy M, Newman-Toker D. Reversible chest tube horner syndrome. J Neuroophthalmol. 2008;28(3):212-3.

49. Kollef MH, Dothager DW. Reversible cardiogenic shock due to chest tube compression of the right ventricle. Chest. 1991;99 (4):976-80.

50. Rahman NM, Singanayagam A, Davies HE, et al. Diagnostic accuracy, safety and utilisation of respiratory physician-delivered thoracic ultrasound. Thorax. 2010;65(5):449-53.

51. Board of the Faculty of Clinical Radiology. Ultrasound training recommendations for Medical and Sugical Specialties. The Royal College of Radiologsts, London. 2004; Available from: http:// www.rcr.ac.uk/docs/radiology/pdf/ultrasound.pdf.

52. Australaisian Society for Ultrasound in Medicine. Certificate in Clinician Performed Ultrasound (CCPU). Available from: http:// www.asum.com.au/newsite/Education.php?p=CCPU.

53. Agarwal PP, Seely JM, Matzinger FR, et al. Pleural mesothelioma: sensitivity and incidence of needle track seeding after image-guided biopsy versus surgical biopsy. Radiology. 2006;241(2):589-94.

54. Lee C, Bayman N, Swindell R, et al. Prophylactic radiotherapy to intervention sites in mesothelioma: a systematic review and survey of UK practice. Lung Cancer. 2009;66(2):150-6.

55. - Davies HE, Musk AW, Lee YC. Prophylactic radiotherapy for pleural puncture sites in mesothelioma: the controversy continues. Curr Opin Pulm Med. 2008;14(4):326-30. A comprehensive review of the current evidence for and against the use of prophylactic radiotherapy in mesothelioma.

56. O'Rourke N, Garcia JC, Paul J, et al. A randomised controlled trial of intervention site radiotherapy in malignant pleural mesothelioma. Radiother Oncol. 2007;84(1):18-22.

57. Bydder S, Phillips M, Joseph DJ, et al. A randomised trial of single-dose radiotherapy to prevent procedure tract metastasis by malignant mesothelioma. Br J Cancer. 2004;91(1):9-10.

58. Boutin C, Rey F, Viallat JR. Prevention of malignant seeding after invasive diagnostic procedures in patients with pleural mesothelioma. A randomized trial of local radiotherapy. Chest. 1995;108 (3):754-8.

59. Janes SM, Rahman NM, Davies RJ, et al. Catheter-tract metastases associated with chronic indwelling pleural catheters. Chest. 2007;131(4):1232-4.

60. Rutledge M, Aronoff D, de Riese W, et al. Management of pleural injuries during retroperitoneal surgical procedures. Int Urol Nephrol. 2007;39(3):717-22.

61. Del Pizzo JJ, Jacobs SC, Bishoff JT, et al. Pleural injury during laparoscopic renal surgery: early recognition and management. J Urol. 2003;169(1):41-4

62. Dondero F, Taille C, Mal H, et al. Respiratory complications: a major concern after right hepatectomy in living liver donors. Transplantation. 2006;81(2):181-6.

63. Kennedy C, McCarthy C, Alken S, et al. Pleuroperitoneal leak complicating peritoneal dialysis: a case series. Int $\mathrm{J}$ Nephrol. 2011;2011:526753. 
64. Hooper C, Lee YC, Maskell N. Investigation of a unilateral pleural effusion in adults: British Thoracic Society Pleural Disease Guideline 2010. Thorax. 2010;65 Suppl 2:ii4-ii17.

65. Light RW, Rogers JT, Moyers JP, et al. Prevalence and clinical course of pleural effusions at 30 days after coronary artery and cardiac surgery. Am J Respir Crit Care Med. 2002;166(12 Pt 1):1567-71.

66. Mamidanna R, Bottle A, Aylin P, et al. Short-term outcomes following open versus minimally invasive esophagectomy for cancer in England: a population-based national study. Ann Surg 2011.
67. Doerr CH, Allen MS, Nichols 3rd FC, et al. Etiology of chylothorax in 203 patients. Mayo Clin Proc. 2005;80(7):867-70.

68. Cafarotti S, Dall'Armi V, Cusumano G, et al. Small-bore wireguided chest drains: safety, tolerability, and effectiveness in pneumothorax, malignant effusions, and pleural empyema. J Thorac Cardiovasc Surg. 2011;141(3):683-7.

69. Collop NA, Kim S, Sahn SA. Analysis of tube thoracostomy performed by pulmonologists at a teaching hospital. Chest. 1997;112(3):709-13.

70. Horsley A, Jones L, White J, et al. Efficacy and complications of small-bore, wire-guided chest drains. Chest. 2006;130(6):1857-63. 